\title{
The Impact of COVID-19 on Inpatient Psychiatry Resident Supervision
}

\author{
William H. Coe ${ }^{1}$ (D) Hun Millard ${ }^{1}$
}

Received: 14 July 2020 / Accepted: 5 October 2020 / Published online: 26 October 2020

(C) Academic Psychiatry 2020

To the Editor:

As an intern during the initial phase of the COVID-19 pandemic, the first author (WC) worked for an entire month on an inpatient adolescent psychiatry unit without ever seeing his attending in person. The pandemic changed everything from workflow, patient care, teaching, and learning. Yet, despite all this, the supervision he received during this month was endorsed to be "some of the best I had all year."

There are numerous articles to date that explore the impact of COVID-19 on resident training and education, particularly within the surgical and procedural subspecialties. Within psychiatry, however, there are significantly fewer articles exploring this topic. Both the American Academy of Geriatric Psychiatry (AAGP) and National Neuroscience Curriculum Initiative (NNCI) have created web-based didactics for residents $[1,2]$, but these are intended to be additive resources and do not directly address changes to clinical supervision or patient care as a result of the pandemic. Other articles discuss rapid advancements in telepsychiatry but do not reflect on the unique challenge of resident supervision on an inpatient setting [3, 4]. Given this, the authors felt it may be helpful to share their experiences in this regard.

Before the pandemic, the 22-bed adolescent unit (for patients ages 13-18) included two clinical teams, each consisting of one attending, one intern, one medical student, and $2-3$ social workers. In the mornings, everyone would meet for "huddle" to discuss new admissions, review potential discharges, and debrief any significant events from overnight before splitting up into their respective teams for patientcentered rounds. The social workers tended to see patients separately given their daily workflow. Attendings, residents, and medical students tended to see patients together, though this occasionally varied depending on learner experience, pa-

William H. Coe

william.coe@yale.edu

Yale School of Medicine, New Haven, CT, USA tient complexity, and other demands on time (such as family meetings, scheduled didactics, and administrative duties). Afternoons were typically reserved for evaluating new patients (done as an entire team), writing progress notes, following up with patients, and engaging in various didactics or learning opportunities.

Once the pandemic hit, the hospital transitioned to "skeleton staffing" with as many clinicians working from home as possible. For two weeks at a time, one social worker and either the intern or attending would be on-site. This meant that while the resident was on the unit, the attending and two social workers were working from home. Morning huddle, rounds, family meetings, etc. all happened by phone or video.

This transition was rapid, chaotic, and not without challenges, but when it came to virtual supervision and learning, this new system seemed to work beautifully in a number of ways. First, it served as a wake-up call. It was impossible to just go through the motions. To provide effective patient care, everyone had to be engaged, in the moment, and hyperfocused on the team dynamic and shared goals.

Second, it opened up and improved lines of communication. Before the pandemic, teams would come together for morning huddle, rounds, and occasional family meetings or new admissions, but spent a majority of their time in separate work rooms. By necessity, this new system required effective teamwork and continuous communication. The threshold for a quick check-in or impromptu teaching session by phone or video was dramatically reduced. And because all of the patients were seen together (albeit virtually), supervisors were able to more consistently and effectively model various clinical skills and techniques, as well as provide residents with immediate feedback on their performance.

Third, this new system allowed social workers to play a more integral role in resident supervision. Residents worked much more closely with the social workers during the height of the pandemic and benefitted greatly from their added perspective and expertise.

Finally, while on-site, residents were the only psychiatrist from their team physically present on the unit (though the 
attending from the sister team was present if needed). As a result, residents had more responsibility than they otherwise would in conducting rounds, managing issues on the unit, and assisting patients in acute distress - all helpful skills for interns to develop before transitioning to second year, taking overnight call, and accepting increased levels of clinical independence.

Yet, despite all this, there were a number of ways in which the pandemic negatively impacted resident supervision and learning. For example, given the reliance on phone and video conferencing, it was much more difficult to observe certain subtleties of the mental status exam or non-verbal communication. Family meetings were hindered by technological issues like spotty audio and weak internet signals. And when at home, it was practically impossible to work with patients on an individual basis.

While this article primarily discusses the strengths of virtual supervision and learning, the authors recognize that others may have different experiences. For example, attendings who provide effective supervision in person may feel restricted in what they can provide learners by phone or video alone, and other attendings who are less invested in resident supervision may further disengage. Similarly, residents who prefer in person supervision may find this new system to be isolating, cumbersome, and detrimental to their learning.

As we seek to establish a new normal for inpatient psychiatry in the time of coronavirus, it is important to reflect on the relative strengths and weaknesses of our adapting systems on patient care and resident supervision. Interestingly, the new system described in this article appears to facilitate learning in a number of ways, namely, by creating greater reliance on teamwork and communication, more consistent role- modeling and feedback, frequent opportunities for interprofessional growth, and increased levels of resident independence. Further advancements in phone and video technology will likely mitigate many of the current shortcomings and greatly enhance patient care and education moving forward.

\section{Compliance with Ethical Standards}

Disclosures On behalf of all authors, the corresponding author states that there is no conflict of interest.

\section{References}

1. Conroy ML, Garcia-Pittman EC, Ali H, Lehmann SW, Yarns BC. The COVID-19 AAGP online trainee curriculum: development and method of initial evaluation. Am J Geriatr Psychiatry [Internet]. 2020. Available from: https://linkinghub.elsevier.com/retrieve/pii/ S1064748120303729

2. Ross DA. Creating a "quarantine curriculum" to enhance teaching and learning during the COVID-19 pandemic. Acad Med [Internet]. 2020. Available from: https://journals.lww.com/10.1097/ACM. 0000000000003424

3. Lin K, Yang BX, Luo D, Liu Q, Ma S, Huang R, et al. The mental health effects of COVID-19 on health care providers in China. Am J Psychiatry. 2020;177(7):635-6 Available from: http://ajp. psychiatryonline.org/doi/10.1176/appi.ajp.2020.20040374.

4. Richards M, DeBonis K. Psychiatric training during a global pandemic: how COVID-19 has affected clinical care, teaching, and trainee well-being. Psychiatr Serv [Internet]. 2020;1-3. Available from: https://psychiatryonline.org/doi/10.1176/appi.ps.202000277

Publisher's Note Springer Nature remains neutral with regard to jurisdictional claims in published maps and institutional affiliations. 